\title{
Somatic Missense Mutations of Histone Variant H3.3 in Central Nervous System Cancers
}

\author{
Burcu Biterge Sut ${ }^{1}$ (D) \\ 'Niğde Ömer Halisdemir University, Faculty of Medicine, Department of Medical Biology, Niğde, Turkey
}

ORCID IDs of the authors: B.B.S. 0000-0001-5756-5756

Please cite this article as: Biterge Sut B. Somatic Missense Mutations of Histone Variant H3.3 in Central Nervous System Cancers. Eur J Biol 2020; 79(2): 75-82. DOI: 10.26650/EurJBiol.2020.0019

\begin{abstract}
Objective: Histone variants are important modulators of chromatin functions. Studies have pointed out that epigenetic factors are often dysregulated in carcinogenesis. Although some cancer-associated mutations of the histone variant $\mathrm{H} 3.3$ have been identified previously, a complete list of H3.3 mutations and their potential effects is yet to be uncovered. Therefore, this study aims to identify the missense mutations of the histone variant $\mathrm{H} 3.3$ in central nervous system (CNS) cancers and to computationally predict their functional consequences on pathogenicity, protein stability and structure.
\end{abstract}

Materials and Methods: A complete set of human H3.3 mutations was acquired from the COSMIC v90 database and missense mutations were selected. The potential effects of these mutations were assessed using PredictSNP2 and FATHMMXF. Structural outcomes were predicted using MUpro and HOPE servers.

Results: We identified 45 unique missense H3.3 substitutions in several tissues including CNS. PredictSNP2 and FATHMM-XF predicted 17 and 42 mutations as deleterious respectively, most of which caused decreased protein stability. Amino acid alterations in CNS cancers were predicted to cause alterations of the 3D structure.

Conclusion: Histone variants play significant roles in epigenetic regulation and are often mutated in cancers. Our results showed that $\mathrm{H} 3.3$ mutations detected in CNS cancers could affect the genomic distribution of post-translational modifications and histone variants, hence dramatically alter the gene expression profile and contribute to carcinogenesis.

Keywords: Epigenetics, histone variant H3.3, mutation analysis, cancer

\section{INTRODUCTION}

Epigenetic regulation is a phenomenon that modulates cellular processes such as proliferation, progression through the cell cycle, transcriptional memory, and DNA damage repair via regulating accessibility of DNA through chromatin condensation (1). The genetic material of eukaryotic organisms exists within the cell as a complex macromolecule called chromatin, consisting of DNA and histone proteins. The packaging of DNA into chromatin dictates differential gene expression patterns which are crucial for the proper functioning of the cell. Perturbations in these processes, as well as transcriptional regulation mechanisms are often associated with complex diseases such as cancer. These regulatory mechanisms are orchestrated by DNA meth- ylation, RNA interference, post-translational histone modifications and incorporation of histone variants into chromatin. Histones are small, basic proteins encoded by several copies of histone genes located within the major histone locus. Canonical histones, namely $\mathrm{H} 2 \mathrm{~A}, \mathrm{H} 2 \mathrm{~B}, \mathrm{H} 3$ and $\mathrm{H} 4$ are strictly synthesized during the S-phase and deposited onto the chromatin in a replication-dependent manner. Histone variants, however, are expressed throughout the cell cycle and incorporated into chromatin in a context-dependent manner (2). Canonical histones and their corresponding variants differ in their amino acid sequence, which affects interactions between histone proteins within the same nucleosome and results in alterations in transcriptional activity. The most commonly studied histone variants 
are centromere specific $\mathrm{H} 3$ variant CENP-A (3); DNA damage site specific H2A.X (4); H2A.Z and H3.3 variants that are commonly found at active transcription sites (5); and macroH2A which is associated with transcriptional repression (6).

Tumors of the brain and the spinal cord, which collectively comprise the central nervous system (CNS), are amongst the most heterogeneous cancer types. The World Health Organization classifies CNS cancers into more than 120 subtypes based on molecular and histopathological characteristics (7). CNS tumors often originate from different cell types, such as astrocytes, glias and meninges. Gliomas and meningiomas are the major subtypes of brain tumors in adults and are rarely seen (8). On the contrary, CNS cancers are the most common solid tumor type in children between the ages of 0-14 (9), 30\% of which is constituted by medulloblastomas (10). Gliomas are graded at four levels depending on the severity, aggressiveness and curability of the disease. Medulloblastoma is the common name for a group of malignant embryonic tumor subtypes that originate from the primitive neuronal cells within the posterior cranial fossa (11). Although the etiology of CNS cancers remains unknown to date, several genetic factors have been associated with increased risk. For instance, a recent study indicated the significant contribution of germline mutations and a genetic disposition to pediatric medulloblastoma (12). Similarly, the mutational status of TP53, BRAF, FGFR1, IDH and TERT as well as the copy number variations of EGFR, CDKN2A/B, PTEN, PDGFRA are often linked with tumor pathogenesis in the CNS $(13,14)$. These genetic variations also serve as powerful molecular tools for cancer subtype characterization.

Studies have shown that several epigenetic factors, including histone variants, are mutated or their activities are dysregulated during cancer pathogenesis. Although previous studies have identified some cancer-associated mutations of the histone variant $\mathrm{H} 3.3$ in chondroblasoma, pediatric sarcoma, giant cell tumor of bone, glioma and medulloblastoma (15-17), a complete list of H3.3 mutations and their potential effects is yet to be uncovered. Therefore, this paper aims to identify H3.3 mutations in CNS cancers and to predict their functional consequences on pathogenicity, protein stability and structure using computational approaches.

\section{MATERIALS AND METHODS}

\section{Retrieval of Somatic Mutations from COSMIC Database}

Somatic mutations of the H3F3A gene (COSMIC gene ID: COSG55679) encoding human $\mathrm{H} 3.3$ were downloaded from the Catalogue of Somatic Mutations in Cancer (COSMIC) database v90 (https://cancer.sanger.ac.uk/cosmic).

\section{Identification of Deleterious Mutations}

The functional consequences and the pathogenicity scores of the H3.3 mutations were predicted using PredictSNP2 (https:// loschmidt.chemi.muni.cz/predictsnp2/) (18) and FATHMM-XF (http://fathmm.biocompute.org.uk/fathmm-xf/index.html) (19) in reference to genome assembly GRCh38/hg38.

\section{Protein Stability Prediction}

The effect of the missense mutations on the stability of the protein was analyzed via MUpro using H3.3 amino acid sequence retrieved from UniProt (ID: P84243), which is based on machine learning methods (http://mupro.proteomics.ics.uci.edu/) (20). The tool provides $84.2 \%$ accuracy.

\section{Determination of 3D Structural Changes}

The structural effects of the nonsynonymous H3.3 mutations, which were commonly encountered in CNS cancers were predicted via HOPE server using H3.3 amino acid sequence retrieved from UniProt (ID: P84243) (https://www3.cmbi.umcn.nl/ hope) (21).

\section{In silico Evaluation of $\mathrm{H} 3.3$ Conservation}

The amino acid sequences of histone H3.3 for Homo sapiens (P84243-1), Mus musculus (P84244-1), Rattus norvegicus (P84245-1), Gallus gallus (P84247-1), Xenopus laevis (Q6PI79-1), Danio rerio (Q6PI20-1), Saccharomyces cerevisiae (P10651-1) and Arabidopsis thaliana (P59169-1) were retrieved from UniProt. In silico evaluation of protein similarity was performed using Clustal Omega (https://www.ebi.ac.uk/Tools/msa/clustalo/) (22). Histone domain structure was determined in accordance with Luger et al. (23).

\section{RESULTS}

\section{Identification of H3.3 Mutations}

COSMIC database analysis over 1012 unique samples showed that $94.2 \%$ of all somatic H3.3 mutations were missense substitutions ( $n=953$ ), most of which were caused by $A>T$ nucleotide change. Furthermore, 2 nonsense substitutions, 8 synonymous substitutions, 2 frameshift deletions, 1 inframe insertion, 1 inframe deletion and 2 uncharacterized mutations were detected. The missense mutations were detected in various tissue types including but not limited to breast, cervix, prostate, upper aerodigestive tract, bone and central nervous system tissues. Table 1 shows a complete list of 45 nonsynonymous mutations identified in $\mathrm{H} 3.3$. Proteins often undergo $\mathrm{N}$-terminal methionine cleavage by methionine aminopeptidase (MAP), which removes the first methionine coded by the start codon (24). Therefore, the starting methionine is not always present in the mature protein. We realized that the locations of the amino acid substitutions identified by the COSMIC database analysis differ from the mature protein by one amino acid, since the COSMIC database did not take the methionine removal into account. The amino acid changes before (detected by COSMIC) and after MAP cleavage are shown in Table 1. For coherence with other studies in the literature, our further analyses were based on the locations of amino acid substitutions after $\mathrm{N}$-terminal methionine cleavage.

\section{Prediction of Pathogenicity and Protein Stability}

The potential effects of somatic H3.3 mutations on carcinogenesis were predicted using two methods. PredictSNP2 calculates an expected accuracy value, which is the consensus classifier for prediction of the effects of nucleotide variants based on 5 different nucleotide-based prediction tools (CADD, DANN, FATHMM, FunSeq2 and GWAVA). PredictSNP2 analysis revealed that 17 
Table 1. Computational predictions of pathogenicity and protein stability for the H3.3 mutations.

\section{PredictSNP2 Analysis FATHMM-XF}

MUpro Analysis

\begin{tabular}{|c|c|c|c|c|c|c|c|}
\hline $\begin{array}{c}\text { CDS } \\
\text { mutation }\end{array}$ & $\begin{array}{l}\text { AA mutation } \\
\text { (COSMIC) }\end{array}$ & $\begin{array}{l}\text { AA mutation } \\
\text { (MAP cleavage) }\end{array}$ & $\begin{array}{l}\text { Pathogenicity } \\
\text { prediction }\end{array}$ & $\begin{array}{l}\text { Expected } \\
\text { accuracy }\end{array}$ & $\begin{array}{l}\text { Pathogenicity } \\
\text { score }\end{array}$ & $\begin{array}{l}\text { Stability } \\
\text { prediction }\end{array}$ & $\begin{array}{l}\text { Confidence } \\
\text { score }\end{array}$ \\
\hline c.7C>T & p.R3C & p.R2C & Neutral & $65 \%$ & 0.580 & Decrease & -0.639 \\
\hline c. $14 A>T$ & p.K5M & p.K4M & Neutral & $63 \%$ & 0.860 & Increase & 0.133 \\
\hline c. $17 A>C$ & p.Q6P & p.Q5P & Neutral & $63 \%$ & 0.941 & Decrease & -0.469 \\
\hline c.18G>C & p.Q6H & p.Q5H & Neutral & $67 \%$ & 0.436 & Decrease & -0.481 \\
\hline c. $25 C>G$ & p.R9G & p.R8G & Neutral & $63 \%$ & 0.913 & Decrease & -1.865 \\
\hline$c .25 C>T$ & p.R9C & p.R8C & Neutral & $63 \%$ & 0.879 & Decrease & -0.853 \\
\hline c.26G>A & p.R9H & p.R8H & Neutral & $63 \%$ & 0.873 & Decrease & -1.566 \\
\hline c.34A>G & p.T12A & p.T11A & Neutral & $65 \%$ & 0.839 & Decrease & -1.544 \\
\hline c.37G $>A$ & p.G13S & p.G12S & Neutral & $63 \%$ & 0.882 & Decrease & -1.347 \\
\hline C. $60 A>C$ & p.Q2OH & p.Q19H & Neutral & $89 \%$ & 0.323 & Decrease & -0.804 \\
\hline c.67A $>C$ & p.T23P & p.T22P & Deleterious & $82 \%$ & 0.940 & Decrease & -1.642 \\
\hline c.76G $>T$ & p.A26S & p.A25S & Neutral & $65 \%$ & 0.878 & Decrease & -1.333 \\
\hline c.82A>G & p.K28E & p.K27E & Neutral & $63 \%$ & 0.874 & Decrease & -0.218 \\
\hline$c .83 A>T$ & p.K28M & p.K27M & Deleterious & $82 \%$ & 0.874 & Decrease & -1.566 \\
\hline$c .84 G>T$ & p.K28N & p.K27N & Neutral & $63 \%$ & 0.559 & Decrease & -0.414 \\
\hline c.86G $>C$ & p.S29T & p.S28T & Neutral & $63 \%$ & 0.863 & Decrease & -0.496 \\
\hline c.98C $>\mathrm{T}$ & p.T33। & p.T32I & Neutral & $63 \%$ & 0.856 & Decrease & -0.256 \\
\hline$c .103 G>T$ & p.G35W & p.G34W & Deleterious & $82 \%$ & 0.920 & Decrease & -0.756 \\
\hline$c .103 \mathrm{G}>\mathrm{A} / \mathrm{C}$ & p.G35R & p. G34R & Deleterious & $87 \%$ & 0.912 & Decrease & -0.739 \\
\hline c.104G $>\mathrm{T}$ & p.G35V & p.G34V & Deleterious & $87 \%$ & 0.917 & Decrease & -0.746 \\
\hline c. $110 A>T$ & p.K37M & p.K36M & Neutral & $63 \%$ & 0.851 & Increase & 0.315 \\
\hline c. $111 \mathrm{G}>\mathrm{T}$ & p.K37N & p.K36N & Neutral & $65 \%$ & 0.687 & Decrease & -0.176 \\
\hline C. $118 C>A$ & p.H40N & p.H39N & Deleterious & $87 \%$ & 0.890 & Increase & 0.148 \\
\hline c.136A>G & p.T46A & p.T45A & Neutral & $63 \%$ & 0.855 & Decrease & -0.494 \\
\hline c.139G >A & p.V47M & p.V46M & Neutral & $63 \%$ & 0.888 & Decrease & -0.013 \\
\hline c. $143 C>A$ & p.A48E & p.A47E & Neutral & $63 \%$ & 0.889 & Decrease & -0.334 \\
\hline c. $148 C>T$ & p.R50C & p.R49C & Deleterious & $87 \%$ & 0.647 & Decrease & -0.127 \\
\hline c.149G >A & p.R50H & p.R49H & Neutral & $65 \%$ & 0.892 & Decrease & -0.768 \\
\hline c. $160 C>T$ & p.R54C & p.R53C & Deleterious & $82 \%$ & 0.910 & Decrease & -1.181 \\
\hline c.168G > T & p.Q56H & p.Q55H & Deleterious & $82 \%$ & 0.577 & Decrease & -1.236 \\
\hline c. $190 \mathrm{C}>\mathrm{T}$ & p.R64C & p.R63C & Neutral & $67 \%$ & 0.909 & Decrease & -1.283 \\
\hline c.218G >A & p.R73Q & p.R72Q & Deleterious & $82 \%$ & 0.869 & Decrease & -0.505 \\
\hline c. $244 \mathrm{G}>\mathrm{A}$ & p.D82N & p.D81N & Deleterious & $87 \%$ & 0.878 & Decrease & -0.620 \\
\hline c. $245 A>T$ & p.D82V & p.D81V & Deleterious & $87 \%$ & 0.881 & Decrease & -0.105 \\
\hline c. $262 \mathrm{G}>\mathrm{T}$ & p.A88S & p.A87S & Deleterious & $82 \%$ & 0.636 & Decrease & -0.748 \\
\hline c.268A $>G$ & p.190V & p.189V & Neutral & $65 \%$ & 0.480 & Decrease & -0.920 \\
\hline C.295G $>A$ & p.A99T & p.A98T & Neutral & $67 \%$ & 0.836 & Decrease & -1.258 \\
\hline c.299A $>G$ & p.Y100C & p.Y99C & Neutral & $63 \%$ & 0.906 & Decrease & -1.230 \\
\hline$c .317 A>T$ & p.E106V & p.E105V & Neutral & $63 \%$ & 0.918 & Decrease & -0.412 \\
\hline c.344C>G & p.A115G & p.A114G & Deleterious & $82 \%$ & 0.924 & Decrease & -1.305 \\
\hline$c .371 A>G$ & p.D124G & p.D123G & Deleterious & $82 \%$ & 0.913 & Decrease & -1.351 \\
\hline c.378G $>T$ & p.Q126H & p.Q125H & Deleterious & $87 \%$ & 0.792 & Decrease & -1.024 \\
\hline$c .385 C>T$ & p.R129C & p.R128C & Neutral & $65 \%$ & 0.926 & Decrease & -0.823 \\
\hline c.386G $>A$ & p.R129H & p.R128H & Neutral & $63 \%$ & 0.912 & Decrease & -1.120 \\
\hline$c .389 \mathrm{G}>\mathrm{A}$ & p.R130H & p.R129H & Deleterious & $82 \%$ & 0.915 & Decrease & -1.542 \\
\hline
\end{tabular}




\section{Distribution of H3.3 mutations across tissues}

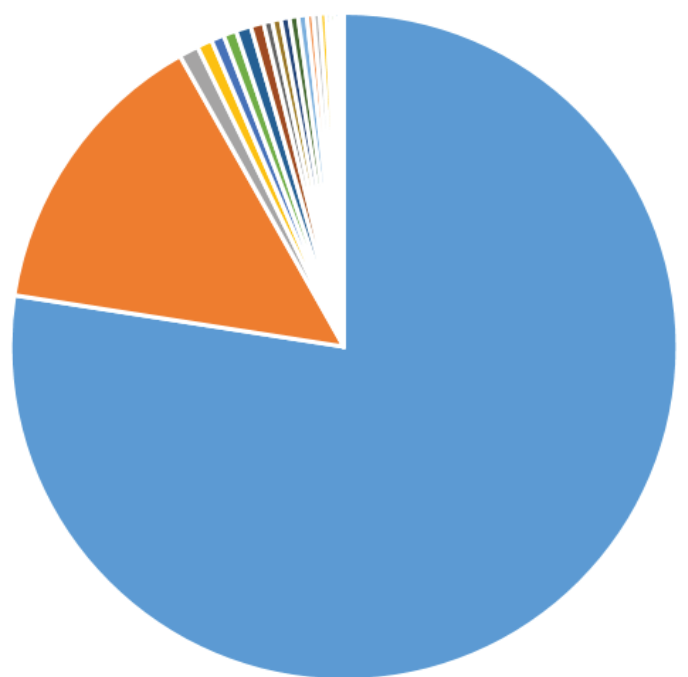

- Central nervous system

- Bone

- Haematopoietic and lymphoid

- Urinary tract

- Breast

- Endometrium

- Large intestine

- Upper aerodigestive tract

- Adrenal gland

- Autonomic ganglia

- Liver

- Stomach

- Thyroid

- Pancreas

- Prostate

- Soft tissue

- Cervix

= Lung

- Penis

- Oesophagus

- Skin

Figure 1. Distribution of the somatic missense mutations of histone variant $\mathrm{H} 3.3$ across tissue types. The majority of the mutations are detected in central nervous system (77\%) and bone (14\%).

out of 45 nucleotide substitutions were deleterious mutations with more than $82 \%$ expected accuracy. Higher percentage values indicate higher confidence; hence, G34R/V, H39N, R49C and D81N/V substitutions had the most confident pathogenicity prediction percentages (87\%).

A second analysis was performed using FATHMM-XF, which produces $\mathrm{p}$-values (pathogenicity scores) between $0-1$ and predicts mutations with $\mathrm{p}>0.5$ as pathogenic/deleterious. FATHMM-XF analysis yielded 42 mutations predicted to be deleterious.

Lastly, the effect of H3.3 mutations on the overall protein stability was predicted using the MUpro tool, which calculates a confidence score, the prediction of the value of energy change (delta delta G), using a machine learning approach, namely the Support Vector Machine. Values $<0$ indicate decreased protein stability, while values $>0$ suggest increased protein stability, showing that most of the mutations caused decreased protein stability with varying confidence scores. Amino acid substitutions with the lowest confidence scores, which are R8G, R8H, $\mathrm{T} 11 \mathrm{~A}, \mathrm{~K} 27 \mathrm{M}$ and $\mathrm{R} 129 \mathrm{H}$, are predicted to result in a greater decrease in protein stability.

\section{H3.3 Mutations in CNS}

Among the analyzed set of unique $\mathrm{H} 3.3$ missense mutations, the majority were identified in CNS (77\%) and bone (14\%) (Figure 1). Therefore, we selected the ones that originated from the CNS as the primary site for further analysis. H3.3 mutations in the CNS namely p.R2C, p.R8H, p.K27M, p.G34R, p.G34R, p.G34W and p.G34V, were mainly derived from brain, cerebral hemisphere, temporal lobe and frontal lobe, while they were also detected to a

Table 2: Somatic missense mutations of histone variant H3.3 in central nervous system (CNS) cancers.

\begin{tabular}{|c|c|c|c|c|}
\hline AA mutation & CDS mutation & Primary tissue & Tissue subtype & Histology \\
\hline p.R2C & c.7C>T & CNS & Brain & Glioma \\
\hline p.R8H & c. $26 \mathrm{G}>\mathrm{A}$ & CNS & Brain & $\begin{array}{c}\text { Glioma, primitive neuroectodermal } \\
\text { tumour-medulloblastoma }\end{array}$ \\
\hline p.K27M & c. $83 \mathrm{~A}>\mathrm{T}$ & CNS & $\begin{array}{l}\text { Brain, cerebral hemisphere, } \\
\text { thalamus, temporal lobe, spinal } \\
\text { cord, posterior fossa, brainstem }\end{array}$ & $\begin{array}{l}\text { Glioma, primitive neuroectodermal } \\
\text { tumour-medulloblastoma }\end{array}$ \\
\hline p.G34R & c. $103 \mathrm{G}>\mathrm{A}$ & CNS & $\begin{array}{l}\text { Brain, cerebral hemisphere, } \\
\text { occipital lobe, frontal lobe, } \\
\text { parietal lobe, temporal lobe }\end{array}$ & $\begin{array}{l}\text { Glioma, primitive neuroectodermal } \\
\text { tumour-medulloblastoma }\end{array}$ \\
\hline p.G34R & c. $103 \mathrm{G}>\mathrm{C}$ & CNS & $\begin{array}{c}\text { Basal ganglia, cerebral hemisphere, } \\
\text { temporal lobe }\end{array}$ & $\begin{array}{c}\text { Glioma, primitive neuroectodermal } \\
\text { tumour-medulloblastoma }\end{array}$ \\
\hline p.G34W & c. $103 \mathrm{G}>\mathrm{T}$ & CNS & Frontal lobe & Glioma \\
\hline p.G34V & c. $104 \mathrm{G}>\mathrm{T}$ & CNS & Brain, frontal lobe & Glioma \\
\hline
\end{tabular}




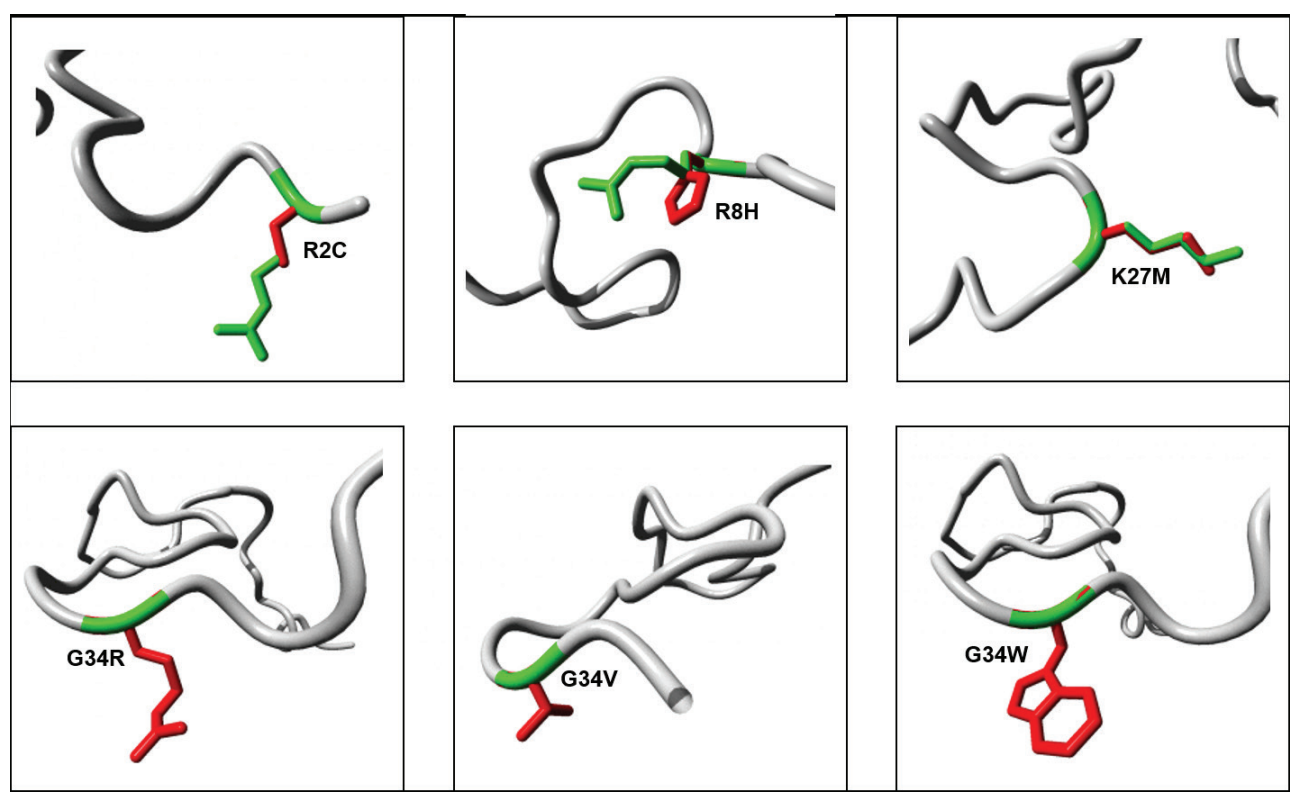

Figure 2. The $3 \mathrm{D}$ constructions depicting structural changes due to missense substitutions R2C, R8H, K27M, G34R, G34V and G34W that are found in glioma. Wild-type and mutated amino acids are indicated in green and red respectively.

lesser degree in thalamus, spinal cord, posterior fossa, brain stem, occipital lobe, parietal lobe, temporal lobe and basal ganglia ( $\mathrm{Ta}$ ble 2). Histologically, the tissues exhibited properties of glioma and primitive neuroectodermal tumour-medulloblastoma.

\section{Analysis of Structural Alterations}

Size, charge, and hydrophobicity are significant features of amino acids which critically differentiate them from one another. Therefore, newly introduced mutant residues often result in structural alterations within the protein. We assessed these potential 3D alterations using the HOPE server (Figure 2). For p.R2C and p.R8H substitutions, the side chains of the two amino acids were significantly different. The mutant residue was smaller and had a different charge when compared to the wild-type protein. p.K27M substitution resulted in a smaller mutant residue, while it was more hydrophobic than the wild-type residue. Substitution of G34 to R, V and W caused bigger and more hydrophobic mutant residues. p.G34R and p.G34W gave rise to the incorpo-

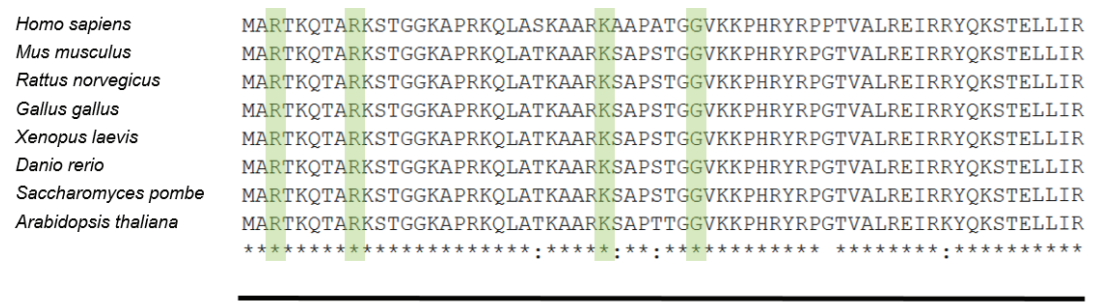

$\mathrm{N}$-terminal tail

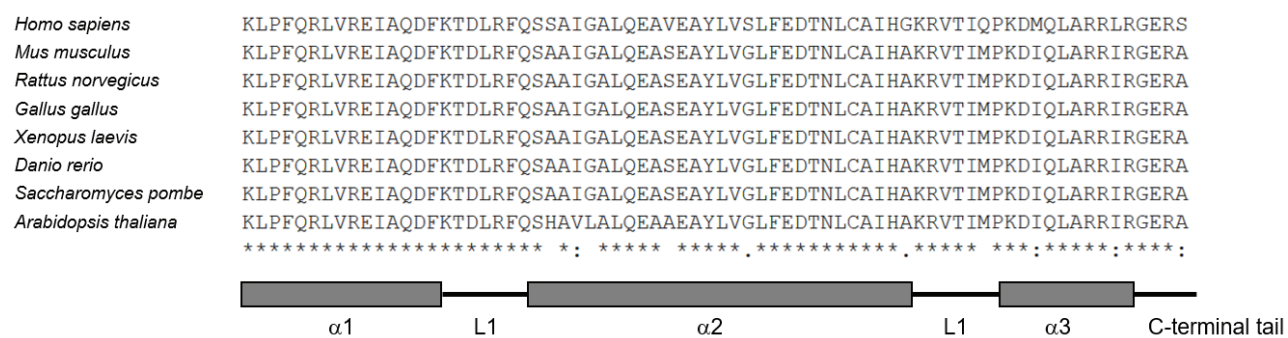

Figure 3. Alignment of histone $\mathrm{H} 3.3$ amino acid sequences in human, mouse, rat, chicken, frog, zebra fish, yeast and plant tissues using Clustal Omega. N- and C-terminal tails and histone fold regions (a1-2-3 helices and L1-2 loops) are indicated. Amino acids R2, R8, K27 and G34 (marked by green) are conserved across species. 
ration of significantly different side chains into the protein. The amino acid sequence alignment of histone H3.3 from human, mouse, rat, chicken, frog, zebra fish, yeast and plant tissues indicated significant conservation across species (Figure 3). All mutant residues given in Figure 2 were located within the highly conserved $\mathrm{N}$-terminal tail of histone $\mathrm{H} 3.3$, which is an important domain for interaction with other molecules.

\section{DISCUSSION}

Histone $\mathrm{H} 3$ has three main variants; while $\mathrm{H} 3.1$ and $\mathrm{H} 3.2$ are classified as the canonical histone H3, H3.3 is the so called "replacement variant" which gets synthesized and incorporated into the chromatin throughout the cell cycle (25). Although the amino acid sequence of H3.3 differs only slightly from the canonical $\mathrm{H} 3$, most of these variant residues lie within structurally important domains that affect its interaction with other histones in the nucleosome and histone chaperones (26). Therefore, nucleosomes containing H3.3 usually confer an open chromatin conformation and are found at active or poised transcription sites that are enriched in tri-methylations of histone $\mathrm{H} 3$ at lysine 4 and $27(27,28)$. Genes encoding epigenetic factors such as histones, their modifiers and chaperons, as well as chromatin remodeling enzymes are often mutated in cancer and are linked with tumorigenesis $(29,30)$. Studies that have previously identified $\mathrm{H} 3.3$ mutations in various cancers mostly focused on the amino acid substitutions at K27, G34 and K36. Mutations in K27 were mainly found in diffuse intrinsic pontine glioma (DIPG), a subtype of pediatric glioma, and were associated with poor prognosis (15-17). G34 substitutions were found in pediatric gliomas located in cerebral hemispheres and giant cell tumors of the bones in young adults $(15,31,32)$. K36 mutations were identified in chondroblastomas and pediatric sarcomas $(17,33)$. In this paper, we identified the whole set of H3.3 mutations in addition those previously identified and characterized their physical and functional properties. Our in-silico analyses showed that most missense mutations of $\mathrm{H} 3.3$ were pathogenic/deleterious and resulted in decreased protein stability.

Among all the somatic missense mutations of histone variant H3.3 retrieved from the COSMIC database across different tissues, the majority were found in the central nervous system cancers. These mutations can potentially act through two mechanisms: either by affecting histone PTMs or altering interactions between histones and their chaperones. Most of these mutation hotspots are functionally critical as they can be post-translationally modified, which is a crucial mechanism for epigenetic regulation of transcriptional activity. $\mathrm{H} 3 \mathrm{~K} 27 \mathrm{me} 3$ is a repressive histone modification located in transcriptionally inactive genes and compact chromatin loci $(34,35)$. Substitution of lysine at position 27 to methionine abolishes this function and results in decreased H3K27me3 levels since methionine cannot be methylated. In line with this, it was previously reported that patients with p.K27M substitutions exhibit globally reduced H3K27me3 levels (36). H3K36me3 is found in actively transcribed gene bodies and missense mutations resulting in p.K36M/N cause reduced H3K36me3 levels (26). Glycine is not directly modified, but it is an amino acid that provides flexibility to the protein, which could be required for its proper functioning; thus, its mutation could result in disrupting this function. Furthermore, due to its proximity to $\mathrm{K} 36$, it is suggested that G34 substitutions could affect the function of H3K36me3 (32). In addition to these previously identified mutations, histone $\mathrm{H} 3$ can be methylated at arginine 2 and 8 by PRMT6 and PRMT5, respectively $(37,38)$. Both of these post-transcriptional modifications are considered as repressive marks (37-40). Substitutions of arginines at positions 2 and 8 would prevent these methylations to take place and alter the transcriptional profile. Therefore, $\mathrm{p} . \mathrm{R} 2 \mathrm{C}$ and $\mathrm{p} . \mathrm{R} 8 \mathrm{H}$ could be as important as K27M and G34R/V/W in CNS tumorigenesis, although they are observed less frequently.

We also showed that all H3.3 mutations detected in CNS cancers introduced structural changes into the protein in varying degrees, which might be affecting its interactions with other proteins, such as histone chaperons. Histones are composed of $\mathrm{N}$ - and/or C-terminal tails and three a-helices connected by two loops, which is called the "histone fold motif" (41). This structure is highly conserved and is significant for interacting with both chromatin modulators and the DNA itself. For instance, histone tails that protrude from the nucleosome often interact with the DNA and contribute to the higher-order chromatin formation (42). Furthermore, histone variant H3.3 is deposited onto chromatin by two main histone chaperon complexes, namely HIRA and DAXX/ATRX (43); and disruption of these interactions would drastically change both the genomic distribution of histone variants and the transcriptional activity. Interestingly, patients with H3.3 mutations are reported to have frequent co-occurring mutations in DAXX and $\operatorname{ATRX}(44,45)$. Almost all patients with mutated H3.3 and DAXX/ATRX also exhibit activation of a mechanism called "alternate lengthening of telomeres", which is a hallmark of cancer (44).

\section{CONCLUSION}

Incorporation of histone variant H3.3 into chromatin, as well as its somatic missense mutations dramatically alter the epigenomic landscape and the gene expression profile of a cell. Previous studies suggested that distinct gene expression patterns (45), transcriptome and interactome profiles (46) are established between tumors carrying different H3.3 mutations. Therefore, H3.3 mutations in CNS cancers are considered both as disruptors of the expression of genes required for brain function and as drivers of tumorigenesis $(44,46)$. In conclusion, histone variants play significant roles in epigenetic regulation. Therefore, mutations in histone variant genes often contribute to carcinogenesis. A better understanding of these cancer-related mutations and their potential effects is useful for future studies.

Peer-review: Externally peer-reviewed.

Author Contributions: Conception/Design of study: B.B.S.; Data Acquisition: B.B.S.; Data Analysis/Interpretation: B.B.S.; Drafting Manuscript: B.B.S.; Critical Revision of Manuscript: B.B.S. 
Conflict of Interest: The authors declare that they have no conflicts of interest to disclose.

Financial Disclosure: There are no funders to report for this submission.

\section{REFERENCES}

1. Kouzarides T. Chromatin modifications and their function. Cell 2007; 6(4): 693-705.

2. Albig W, Doenecke D. The human histone gene cluster at the D6S105 locus. Hum Genet 1997; 101: 284-94.

3. Yoda K, Ando S, Morishita S, Houmura K, Hashimoto K, Takeyasu K, et al. Human centromere protein A (CENP-A) can replace histone $\mathrm{H} 3$ in nucleosome reconstitution in vitro. Proc Natl Acad Sci USA 2000; 97: 7266-71.

4. Rogakou EP, Boon C, Redon C, Bonner WM. Megabase chromatin domains involved in DNA double-strand breaks in vivo. J Cell Biol 1999; 146: 905-16.

5. Thakar A, Gupta P, Ishibashi T, Finn R, Silva-Moreno B, Uchiyama S, et al. $\mathrm{H} 2 \mathrm{~A} . \mathrm{Z}$ and $\mathrm{H} 3.3$ histone variants affect nucleosome structure: biochemical and biophysical studies. Biochemistry 2009; 48(46): 10852-7.

6. Chakravarthy S, Gundimella SK, Caron C, Perche PY, Pehrson JR, Khochbin S, et al. Structural characterization of the histone variant macroH2A. Mol Cell Biol 2005; 25: 7616-24.

7. Louis DN, Perry A, Reifenberger G, von Deimling A, Figarella-Branger D, Cavenee WK, et al. The 2016 World Health Organization classification of tumors of the central nervous system: a summary. Acta Neuropathol 2016; 131(6): 803-20. doi: 10.1007/s00401-016-1545-1.

8. McNeill KA. Epidemiology of brain tumors. Neurol Clin 2016; 34(4): 981-98. http://dx.doi.org/10.1016/j.ncl.2016.06.014.

9. Ostrom QT, de Blank PM, Kruchko C, Petersen CM, Liao P, Finlay JL, et al. Alex's Lemonade Stand Foundation infant and childhood primary brain and central nervous system tumors diagnosed in the United States in 2007-2011. Neuro Oncol 2015; 16(Suppl 10): x1-36.

10. Ajeawung NF, Wang HY, Gould P, Kamnasaran D. Advances in molecular targets for the treatment of medulloblastomas. Clin Invest Med 2012; 35(5): E246.

11. Coluccia D, Figuereido C, Isik S, Smith C, Rutka JT. Medulloblastoma: tumor biology and relevance to treatment and prognosis paradigm. Curr Neurol Neurosci Rep 2016; 16(5): 43. doi: 10.1007/ s11910-016-0644-7.

12. Waszak SM, Northcott PA, Buchhalter I, Robinson GW, Sutter C, Groebner S, et al. Spectrum and prevalence of genetic predisposition in medulloblastoma: a retrospective genetic study and prospective validation in a clinical trial cohort. Lancet Oncol 2018; 19(6): 785-98. doi: 10.1016/S1470-2045(18)30242-0.

13. Park SH, Won J, Kim SI, Lee Y, Park CK, Kim SK, et al. Molecular testing of brain tumor. J Pathol Transl Med 2017; 51(3): 205-23. doi:10.4132/jptm.2017.03.08.

14. Aldape K, Zadeh G, Mansouri S, Reifenberger G, von Deimling A. Glioblastoma: pathology, molecular mechanisms and markers. Acta Neuropathol 2015; 129: 829-48.

15. Khuong-Quang DA, Buczkowicz P, Rakopoulos P, Liu XY, Fontebasso AM, Bouffet E et al. K27M mutation in histone H3.3 defines clinically and biologically distinct subgroups of pediatric diffuse intrinsic pontine gliomas. Acta Neuropathol 2012; 124(3): 439-47. doi:10.1007/s00401-012-0998-0.

16. Wan YCE, Liu J, Chan KM. Histone H3 mutations in cancer. Curr Pharmacol Rep 2018; 4(4): 292-300. doi: 10.1007/s40495-0180141-6.
17. Weinberg DN, Allis CD, Lu C. Oncogenic mechanisms of histone H3 mutations. Cold Spring Harb Perspect Med 2017; 3: 7(1). pii: a026443. doi: 10.1101/cshperspect.a026443.

18. Bendl J, Musil M, Štourač J, Zendulka J, Damborský J, Brezovský J. PredictSNP2: a unified platform for accurately evaluating SNP effects by exploiting the different characteristics of variants in distinct genomic regions. PLoS Comput Biol 2016; 12(5): e1004962. doi: 10.1371/journal.pcbi.1004962.

19. Rogers MF, Shihab HA, Mort M, Cooper DN, Gaunt TR, Campbell C. FATHMM-XF: accurate prediction of pathogenic point mutations via extended features. Bioinformatics 2018; 34(3): 511-3. doi: 10.1093/bioinformatics/btx536.

20. Cheng J, Randall A, Baldi P. Prediction of protein stability changes for single-site mutations using support vector machines. Proteins 2006; 62(4): 1125-32.

21. Venselaar H, Te Beek TA, Kuipers RK, Hekkelman ML, Vriend G. Protein structure analysis of mutations causing inheritable diseases. An e-Science approach with life scientist friendly interfaces. BMC Bioinformatics 2010; 11: 548. doi: 10.1186/1471-2105-11-548.

22. Sievers F, Wilm A, Dineen D, Gibson TJ, Karplus K, Li W, et al. Fast, scalable generation of high-quality protein multiple sequence alignments using Clustal Omega. Mol Syst Biol 2011; 7: 539. doi: 10.1038/msb.2011.75.

23. Luger K, Mäder AW, Richmond RK, Sargent DF, Richmond TJ. Crystal structure of the nucleosome core particle at $2.8 \mathrm{~A}$ resolution. Nature 1997; 389(6648): 251-60. doi: 10.1038/38444.

24. Frottin F, Martinez A, Peynot P, Mitra S, Holz RC, Giglione C, et al. The proteomics of $\mathrm{N}$-terminal methionine cleavage. Mol Cell Proteomics 2006; 5(12): 2336-49.

25. Hake SB, Garcia BA, Duncan EM, Kauer M, Dellaire G, Shabanowitz $J$, et al. Expression patterns and post-translational modifications associated with mammalian histone $\mathrm{H} 3$ variants. J Biol Chem 2006; 281(1): 559-68.

26. Lan F, Shi Y. Histone H3.3 and cancer: a potential reader connection. Proc Natl Acad Sci USA 2015; 112(22): 6814-9. doi: 10.1073/ pnas.1418996111.

27. Chen P, Zhao J, Wang $\mathrm{Y}$, Wang $\mathrm{M}$, Long $\mathrm{H}$, Liang $\mathrm{D}$, et al. H3.3 actively marks enhancers and primes gene transcription via opening higher-ordered chromatin. Genes Dev 2013; 27(19): 2109-24.

28. Delbarre $E$, Jacobsen BM, Reiner AH, Sørensen AL, Küntziger $T$, Collas P. Chromatin environment of histone variant $\mathrm{H} 3.3$ revealed by quantitative imaging and genome-scale chromatin and DNA immunoprecipitation. Mol Biol Cell 2010; 21(11): 1872-84. doi: 10.1091/mbc.E09-09-0839.

29. Dawson MA, Kouzarides T. Cancer epigenetics: from mechanism to therapy. Cell 2012; 150(1): 12-27. doi: 10.1016/j.cell.2012.06.013.

30. Suvà $M L$, Riggi $N$, Bernstein BE. Epigenetic reprogramming in cancer. Science 2013; 339(6127): 1567-70. doi: 10.1126/science.1230184.

31. Sturm D, Witt H, Hovestadt V, Khuong-Quang DA, Jones DT, Konermann $\mathrm{C}$, et al. Hotspot mutations in H3F3A and IDH1 define distinct epigenetic and biological subgroups of glioblastoma. Cancer Cell 2012; 22(4): 425-37. doi: 10.1016/j.ccr.2012.08.024.

32. Kallappagoudar S, Yadav RK, Lowe BR, Partridge JF. Histone H3 mutations--a special role for $\mathrm{H} 3.3$ in tumorigenesis? Chromosoma 2015; 124(2): 177-89. doi: 10.1007/s00412-015-0510-4.

33. Behjati S, Tarpey PS, Presneau N, Scheipl S, Pillay N, Van Loo P, et al. Distinct H3F3A and H3F3B driver mutations define chondroblastoma and giant cell tumor of bone. Nat Genet 2013; 45(12): 1479-82. doi: 10.1038/ng.2814.

34. Cao R, Zhang $Y$. The functions of $E(Z) / E Z H 2$-mediated methylation of lysine 27 in histone H3. Curr Opin Genet Dev 2004;14(2): 155-64. 
35. Black JC, Van Rechem C, Whetstine JR. Histone lysine methylation dynamics: establishment, regulation, and biological impact. Mol Cell 2012; 48(4): 491-507. doi: 10.1016/j.molcel.2012.11.006.

36. Bender S, Tang Y, Lindroth AM, Hovestadt V, Jones DT, Kool M, et al. Reduced H3K27me3 and DNA hypomethylation are major drivers of gene expression in $\mathrm{K} 27 \mathrm{M}$ mutant pediatric high-grade gliomas. Cancer Cell 2013; 24(5): 660-72. doi: 10.1016/j.ccr.2013.10.006.

37. Guccione E, Bassi C, Casadio F, Martinato F, Cesaroni M, Schuchlautz $\mathrm{H}$, et al. Methylation of histone H3R2 by PRMT6 and H3K4 by an MLL complex are mutually exclusive. Nature 2007; 449: 933-7.

38. Pal S, Vishwanath SN, Erdjument-Bromage H, Tempst P, Sif S. Human SWI/SNF-associated PRMT5 methylates histone $\mathrm{H} 3$ arginine 8 and negatively regulates expression of ST7 and NM23 tumor suppressor genes. Mol Cell Biol 2004; 24: 9630-45.

39. Hyllus D, Stein C, Schnabel K, Schiltz E, Imhof A, Dou Y, et al. PRMT6-mediated methylation of R2 in histone $\mathrm{H} 3$ antagonizes $\mathrm{H} 3$ K4 trimethylation. Genes Dev 2007; 21: 3369-80.

40. Wang L, Pal S, Sif S. Protein arginine methyltransferase 5 suppresses the transcription of the RB family of tumor suppressors in leukemia and lymphoma cells. Mol Cell Biol 2008; 28: 6262-77.

41. Arents G, Moudrianakis EN. The histone fold: a ubiquitous architectural motif utilized in DNA compaction and protein dimerization. Proc Natl Acad Sci USA 1995; 92(24): 11170-4. doi: 10.1073/ pnas.92.24.11170.
42. Iwasaki S, Iwasaki W, Takahashi M, Sakamoto A, Watanabe C, Shichino $Y$, et al. The translation inhibitor rocaglamide targets a bimolecular cavity between elF4A and polypurine RNA. Mol Cell 2019; 73(4): 738-748.e9. doi: 10.1016/j.molcel.2018.11.026.

43. Goldberg AD, Banaszynski LA, Noh KM, Lewis PW, Elsaesser SJ, Stadler S, et al. Distinct factors control histone variant H3.3 localization at specific genomic regions. Cell 2010; 140(5): 678-91. doi: 10.1016/j.cell.2010.01.003.

44. Yuen BT, Knoepfler PS. Histone H3.3 mutations: a variant path to cancer. Cancer Cell 2013; 24(5): 567-74. doi: 10.1016/j. ccr.2013.09.015.

45. Schwartzentruber J, Korshunov A, Liu XY, Jones DT, Pfaff E, Jacob K, et al. Driver mutations in histone $\mathrm{H} 3.3$ and chromatin remodelling genes in paediatric glioblastoma. Nature 2012; 482(7384): 226-31. doi: 10.1038/nature10833.

46. Lim J, Park JH, Baude A, Fellenberg J, Zustin J, Haller F, et al. Transcriptome and protein interaction profiling in cancer cells with mutations in histone H3.3. Sci Data 2018; 5: 180283. doi: 10.1038/ sdata.2018.283. 